\title{
Association of Primary Sarcopenia with Serum MMP2, TIMP2 Levels, and MMP2/TIMP2 Ratio
}

\author{
(D) Veysel Suzan1, (1) Bahar Bektan Kanat1, (1) Hakan Yavuzer1, (1) Ibrahim Murat Bolayırı², (D) Alper Döventaş1, \\ (1) Deniz Suna Erdinçler 1 \\ 1istanbul University-Cerrahpaşa, Cerrahpaşa Faculty of Medicine, Department of Internal Medicine, Clinic of Geriatric Medicine, Istanbul, Turkey \\ 2istanbul University-Cerrahpaşa, Cerrahpaşa Faculty of Medicine, Department of Biochemistry, İstanbul, Turkey
}

\begin{abstract}
Objective: Our aim is to evaluate the association of serum matrix metalloproteinase (MMP2), tissue inhibitor of metalloproteinase (TIMP2) levels, MMP2/TIMP2 ratio, SARC-F with primary sarcopenia, and to investigate the coexistence of primary sarcopenia and osteoporosis in geriatric population.
\end{abstract}

Materials and Methods: A total of 80 geriatric patients (41 sarcopenic patients) were included in the study. Patients with secondary sarcopenia were excluded. By SARC-F questionnaire, patients who were at risk for sarcopenia were found as mentioned by EWGSOP2. Serum MMP2 and TIMP2 levels were analyzed by ELISA method. Dual energy X-ray absorptiometry (DEXA) was used for the diagnosis of osteoporosis.

Results: The SARC-F score of the sarcopenia group was statistically significantly higher and the incidence of osteoporosis was higher in this group than the group without sarcopenia ( $p<0.001, p=0.006$, respectively). There was no significant difference between the sarcopenic and non-sarcopenic groups in terms of gender, age, serum MMP2, TIMP2 and MMP2/TIMP2 ratio ( $p=0.959, p=0.182, p=0.366, p=0.225, p=0.641$ respectively). In the multivariate regression analysis it was determined that SARC-F and osteoporosis had a significant effect on sarcopenia $[p<0.001$, odds ratio (OR) (95\%) confidence interval (CI) 1.735 (1.292-2.330); $p=0.006$, OR (95\%) Cl 4.976 (1.590-15.572) respectively]. The area under ROC curve (AUC) of SARC-F and MMP2/TIMP2 ratio was 0.771 and 0.530 ( $p<0.001,95 \% \mathrm{Cl} 0.665-0.877 ; \mathrm{p}=0.641, \% 95 \mathrm{Cl} 0.403-0.658$, respectively). Sensitivity and specificity for SARC-F score $\geq 4$ were $58.5 \%$ and $87.2 \%$, respectively.

Conclusion: Our study supports the usage of SARC-F in finding of primary sarcopenia cases. We have revealed that serum MMP2, TIMP2 level and MMP2/TIMP2 ratio are not suitable markers for diagnosing sarcopenia. Since the coexistence of osteoporosis and sarcopenia is common in the elderly, they should be considered together.

Keywords: Sarcopenia, MMP2, TIMP2, SARC-F, osteoporosis

\section{Introduction}

Sarcopenia is a complex and multifactorial disease characterized with a decrease in muscle mass and muscle strength, that causes decreased mobility, increased risk of falling, decreased quality of life and ultimately increased mortality $(1,2)$. Sarcopenia is defined as a decrease in skeletal muscle mass as well as decreased muscle strength or poor muscle performance. While age-related sarcopenia without a specific cause is called primary sarcopenia, sarcopenia that develops in the presence of a significant factor other than aging or in addition to aging is called secondary sarcopenia (3). In line with the recommendations of EWGSOP2, the SARC-F questionnaire is applied to individuals at risk for sarcopenia, and those with high $(\geq 4)$ scores are considered to be at risk for sarcopenia (4). Osteoporosis is a common disease that causes an increased risk of fracture as a result of decreased bone mass and deterioration in microarchitecture. Both sarcopenia and osteoporosis are associated with morbidity, and there are studies suggesting a relationship between these two diseases. There are studies reporting that osteoporosis and sarcopenia

Address for Correspondence: Veysel Suzan, İstanbul University-Cerrahpaşa, Cerrahpaşa Faculty of Medicine, Department of Internal Medicine, Clinic of Geriatric Medicine, İstanbul, Turkey

Phone: +90 5323341581 E-mail: veysel.suzan@hotmail.com ORCID: orcid.org/0000-0001-5741-9820

Received: 12.10.2021 Accepted: 15.12.2021

Cite this article as: Suzan V, Bektan Kanat B, Yavuzer H, Bolayırlı IM, Döventaş A, Erdinçler DS. Association of Primary Sarcopenia with Serum MMP2, TIMP2 Levels, and MMP2/TIMP2 Ratio. Eur J Geriatr Gerontol 2022;4(2):46-51

๑Copyright 2022 by the Academic Geriatrics Society / European Journal of Geriatrics and Gerontology published by Galenos Publishing House. 
come from common genetics (5). According to the mechanostat hypothesis expressing that muscle and bone affect each other, muscle contraction is thought to stimulate bone osteogenesis with a mechanical effect (6).

Matrix metalloproteinases (MMPs) are proteases that are responsible for the degradation of extracellular matrix proteins and play a role in many biological and physiological processes such as organogenesis, growth and tissue regeneration. Although the release and activation of MMPs from healthy adult tissue is limited, their levels increase in pathologies that cause tissue destruction (7). The activity of MMPs is regulated in tissue by "Tissue inhibitors of metalloproteinases (TIMPs)". TIMPs maintain homeostasis and tissue integrity by controlling MMP enzyme activity and MMP/TIMP balance. The shift of this balance in the direction of MMP activity causes the destruction of the matrix and the occurrence of pathophysiological events. TIMPs show specificity according to MMP species, and MMP-2 is inhibited by TIMP-2 (8).

As for the effects of MMPs on muscle tissue, MMPs break down the extracellular matrix in skeletal muscle, regulate migration, differentiation and regeneration of muscle cells and thus play an important role in the homeostasis of myofibers (9). Studies have clearly demonstrated the importance of MMP2 in myoblast migration and fusion $(10,11)$. With the discovery of this, the role of MMP2, its inhibitor TIMP2 and the balance between them in muscle diseases began to be investigated.

Although many methods are used for the diagnosis of sarcopenia, a practical method has not been found yet. In this study, our aim is to investigate the usability of serum MMP2, TIMP2 level and MMP2/TIMP2 ratio as a markers for diagnosis in addition to the diagnostic methods we use for sarcopenia and to evaluate the relationship between sarcopenia and osteoporosis.

\section{Materials and Methods}

\section{Population and setting}

Forty-one sarcopenic patients followed up our geriatric outpatient clinic (female 25/male 16) and thirty nine control patients (female 24/male 15) were recruited for this study. In addition, chronic diseases and geriatric syndromes of individuals with sarcopenia were documented and matched with control group patients. Patients with probable secondary sarcopenia (being bed bound, advanced organ failure, malignancy, proteinenergy malnutrition, HIV infection, chronic inflammatory disease, rheumatoid arthritis, malabsorption, steroid use) and active infection were excluded from the study.

\section{Sarcopenia and osteporosis diagnosis}

EWGSOP2 criteria were considered for the diagnosis of sarcopenia and cut-off values (4). A hand dynamometer was used for muscle strength measurement. The measurement was made while the patients in sitting position, the elbow flexed to 90 degrees, and the wrist in neutral position. Muscle strength was measured for three times for both hands and one minute rest period was left between measurements. The highest measurement was recorded as the handgrip strength. For a positive handgrip strength test, cut-off value was accepted as $27 \mathrm{~kg}$ for men and under $16 \mathrm{~kg}$ for women. Bioelectrical impedance analyzer (BIA) device was used to measure skeletal muscle mass index (SMMI) in $\mathrm{kg} / \mathrm{m}^{2}$ after 12 hours of fasting and its cut-off value was $<7.0 \mathrm{~kg} /$ $\mathrm{m}^{2}$ in males and $<5.5 \mathrm{~kg} / \mathrm{m}^{2}$ in females. A 6 meters long area was determined in the corridor to evaluate physical activity performance. The first 1 meter was determined as acceleration zone, the next 4 meters as timing area and the last one meter as the deceleration zone. The walking speed of each patient was recorded. Gait speed cut-off value was accepted as $\leq 0.8 \mathrm{~m} / \mathrm{s}$. Dual energy $\mathrm{X}$-ray absorptiometry (DEXA) was used for the diagnosis of osteoporosis. Femur and vertebral measurements were taken into account in the diagnosis of osteoporosis. T-score below -2.5 standard deviation (SD) in these measurements was accepted as osteoporosis. While the femoral neck and total score were taken into account when interpreting the femur measurements, total (Lumbar 1-Lumbar 4) or at least two vertebral scores were used in the evaluation of vertebra measurements $(12,13)$.

\section{Biochemical analysis}

For MMP2 and TIMP2 levels, blood was drawn from the antecubital vein between 08:00 and 9:00 a.m. after fasting for at least 8 hours from all participants. The blood was first transferred into an $8 \mathrm{~mL}$ tube without any additives or gel and centrifuged for 15 minutes. The kit inserts were followed for the analytes planned to be examined in our study. The preanalytical process was carried out by geriatric and biochemistry physicians.

\section{Statistics}

Chi-square or Fisher's Exact tests were applied for categorical variables. Student's t-test or Mann-Whitney U test was used for continuous variables. The relationship between sarcopenia and age, gender, MMP2, TIMP2, MMP2/TIMP2 ratio, SARC-F score, and osteoporosis were analyzed by univariate logistic regression (LR) method. Stepwise multivariate LR method was applied to the significant values after univariate LR analysis. ROC analysis was performed to determine the importance of MMP2/TIMP2 ratio and the SARC- $F$ in the diagnosis of sarcopenia. $P<0.05$ was considered significant. Statistical Package for the Social Sciences- 21.0 was used for the analysis of this clinical data.

\section{Results}

In the study, 41 sarcopenic and 39 non-sarcopenic geriatric patients were included. Women constituted $61 \%$ of both patient groups. In terms of age (mean \pm standard deviation), 
no statistically significant difference was found between the group with sarcopenia $(77.65 \pm 5.96)$ and the group without $(75.76 \pm 6.96)(p=0.182)$. While there were $23(56 \%)$ individuals with osteoporosis in the sarcopenic group, there were $10(25 \%)$ individuals in the non-sarcopenic group, and this difference was statistically significant $(p<0.001)$. Total lumbar, total femur, femur neck T-score and bone mineral density (BMD) values were significantly lower in the sarcopenic group compared to the non-sarcopenic group ( $p=0.002, p=0.006, p=0.043$ and $p=0.008$, $p=0.008, p=0.028$; respectively). Considering the SARC-F score (mean \pm standard deviation), the score of the group with sarcopenia $(4.04 \pm 2.35)$ was statistically significantly higher than the group without sarcopenia $(1.87 \pm 1.83)(p<0.001)$. The sarcopenic group had statistically significant lower grip strength, muscle mass, and gait speed $(p<0.001, p<0.001$, $p=0.006$, respectively). There was no significant difference between the two groups in terms of serum MMP2 and TIMP2 $(p=0.366, p=0.225$, respectively). Considering MMP2/TIMP2 ratio, the median of the group with sarcopenia was 10.34 , while it was 10.07 in the group without sarcopenia, and this difference was not statistically significant $(p=0.641)$. These comparisons are given in Table 1.

In univariate logistic regression, no statistical correlation was found between sarcopenia and gender, age, serum MMP2, TIMP2 and MMP2/TIMP2 ratio (respectively $p=0.959, p=0.194, p=0.720$, $p=0.570, p=0.441)$. On the other hand, a significant correlation was found between sarcopenia, and SARC- $F(p<0.001)$ and osteoporosis $(\mathrm{p}=0.007)$. Multivariate logistic regression analysis was performed to these parameters and the results showed that SARC-F and osteoporosis had a significant effect on sarcopenia [ $p<0.001$, OR (95\%) Cl 1.735 (1.292-2.330); $p=0.006$, OR (95\%) Cl 4.976 (1.590-15.572) respectively] (Table 2).

Area under ROC curve (AUC) of SARC-F and MMP2/TIMP2 ratio was 0.771 and 0.530 (Figure 1). Sensitivity and specificity for SARC-F score $\geq 4$ were $58.5 \%$ and $87.2 \%$, respectively.

\section{Discussion}

Sarcopenia in the elderly, generally accepted as an inevitable part of aging, is attracting more and more attention due to its great impact on morbidity, mortality and health costs. According to EWGSOP2, sarcopenia represents a loss of muscle mass and quality with a decrease in muscle strength. Usage of the rapid screening test, SARC-F, which is a self-administered questionnaire was recommended by EWGSOP2 to identify individuals at risk for sarcopenia (4). It is a simple, inexpensive and non-invasive method. After the recommendation about usage of SARC-F by EWGSOP2, many translation and validation studies have been done. Polish and Spanish version of SARC-F was studied and recommended for assessing sarcopenia in everyday practice $(14,15)$. In a study conducted with Danish population, SARC-F
Table 1. Demographic characteristics, grip strength, muscle mass, walking speed and laboratory findings of sarcopenia and no sarcopenia groups

\begin{tabular}{|c|c|c|c|}
\hline & Sarcopenia & No sarcopenia & p \\
\hline Number of patients & 41 & 39 & \\
\hline Gender (female/male) & $25 / 16$ & $24 / 15$ & 0.959 \\
\hline Age & $77.65 \pm 5.96$ & $75.76 \pm 6.96$ & 0.182 \\
\hline SARC-F & $4.04 \pm 2.35$ & $1.87 \pm 1.83$ & $<0.001$ \\
\hline Grip strength & $16.09 \pm 5.63$ & $21.76 \pm 6.12$ & $<0.001$ \\
\hline Muscle mass (SMMI) & $5.73 \pm 0.78$ & $6.44 \pm 0.67$ & $<0.001$ \\
\hline Gait speed & $0.68 \pm 0.33$ & $0.83 \pm 0.27$ & 0.006 \\
\hline MMP2 (ng/mL) & $\begin{array}{l}227.7 \\
(196.3-503.4)\end{array}$ & $\begin{array}{l}260.5 \\
(213.1-726.3)\end{array}$ & 0.366 \\
\hline TIMP2 (ng/mL) & $\begin{array}{l}25.0 \\
(20.5-47.6)\end{array}$ & $\begin{array}{l}30.1 \\
(22.3-61.4)\end{array}$ & 0.225 \\
\hline MMP2/TIMP2 & $\begin{array}{l}10.34 \\
(8.10-12.65) \\
\end{array}$ & $\begin{array}{l}10.07 \\
(7.07-12.48) \\
\end{array}$ & 0.641 \\
\hline Osteoporosis (yes/no) & $23 / 18$ & $10 / 29$ & 0.006 \\
\hline Femur total, T-score & $-1.6 \pm 0.9$ & $-0.9 \pm 0.8$ & 0.006 \\
\hline Femur total, BMD $\left(\mathrm{gr} / \mathrm{cm}^{2}\right)$ & $0.681 \pm 0.158$ & $0.779 \pm 0.136$ & 0.008 \\
\hline Femur neck, T-score & $-1.8 \pm 0.7$ & $-1.4 \pm 0.7$ & 0.043 \\
\hline Femur neck, BMD (gr/ $\left./ \mathrm{cm}^{2}\right)$ & $0.643 \pm 0.095$ & $0.705 \pm 0.115$ & 0.028 \\
\hline Lomber total, T-score & $-1.9 \pm 1.0$ & $-1.2 \pm 0.9$ & 0.002 \\
\hline $\begin{array}{l}\text { Lomber total, BMD } \\
\left(\mathrm{gr} / \mathrm{cm}^{2}\right)\end{array}$ & $0.817 \pm 0.108$ & $0.880 \pm 0.093$ & 0.008 \\
\hline \multicolumn{4}{|c|}{$\begin{array}{l}\text { MMP: Matrix metalloproteinase, TIMP: Tissue inhibitor of metalloproteinase, SMMI: } \\
\text { Skeletal muscle mass index, BMD: Bone mineral density, data are shown as mean } \pm \\
\text { standard deviation or median (interquartile intervals), statistically significant } p \text {-values } \\
\text { are indicated as bold }\end{array}$} \\
\hline
\end{tabular}

Table 2. Univariate and stepwise multivariate LR analysis of prediction of sarcopenia

\begin{tabular}{|c|c|c|c|c|}
\hline & \multicolumn{2}{|l|}{ Univariate LR } & \multicolumn{2}{|c|}{$\begin{array}{l}\text { Stepwise multivariate } \\
\text { LR }\end{array}$} \\
\hline & $\begin{array}{l}\text { Odds ratio } \\
(95 \% \mathrm{Cl})\end{array}$ & p & $\begin{array}{l}\text { Odds ratio } \\
(95 \% \mathrm{Cl})\end{array}$ & $\mathbf{p}$ \\
\hline $\begin{array}{l}\text { Female } \\
\text { Male }\end{array}$ & $\begin{array}{l}1 \\
1.024 \\
(0.416-2.519)\end{array}$ & 0.959 & & \\
\hline Age & $\begin{array}{l}1.047 \\
(0.977-1.122)\end{array}$ & 0.194 & & \\
\hline SARC-F & $\begin{array}{l}1.635 \\
(1.264-2.115)\end{array}$ & $<0.001$ & $\begin{array}{l}1.735 \\
(1.292-2.330)\end{array}$ & $<0.001$ \\
\hline $\begin{array}{l}\text { No osteoporosis } \\
\text { osteoporosis }\end{array}$ & $\begin{array}{l}1 \\
3.706 \\
(1.437-9.554)\end{array}$ & 0.007 & $\begin{array}{l}4.976 \\
(1.590-15.572)\end{array}$ & 0.006 \\
\hline MMP2 (ng/mL) & $\begin{array}{l}1.000 \\
(0.999-1.000)\end{array}$ & 0.720 & & \\
\hline TIMP2 (ng/mL) & $\begin{array}{l}0.998 \\
(0.991-1.005)\end{array}$ & 0.570 & & \\
\hline $\begin{array}{l}\text { MMP2/TIMP2 } \\
\text { ratio }\end{array}$ & $\begin{array}{l}1.051 \\
(0.926-1.193)\end{array}$ & 0.441 & & \\
\hline \multicolumn{5}{|c|}{$\begin{array}{l}\text { MMP: Matrix metalloproteinase, TIMP: Tissue inhibitor of metalloproteinase, LR: } \\
\text { Logistic regression, Cl: Confidence interval, Statistically significant p-values are } \\
\text { indicated as bold }\end{array}$} \\
\hline
\end{tabular}




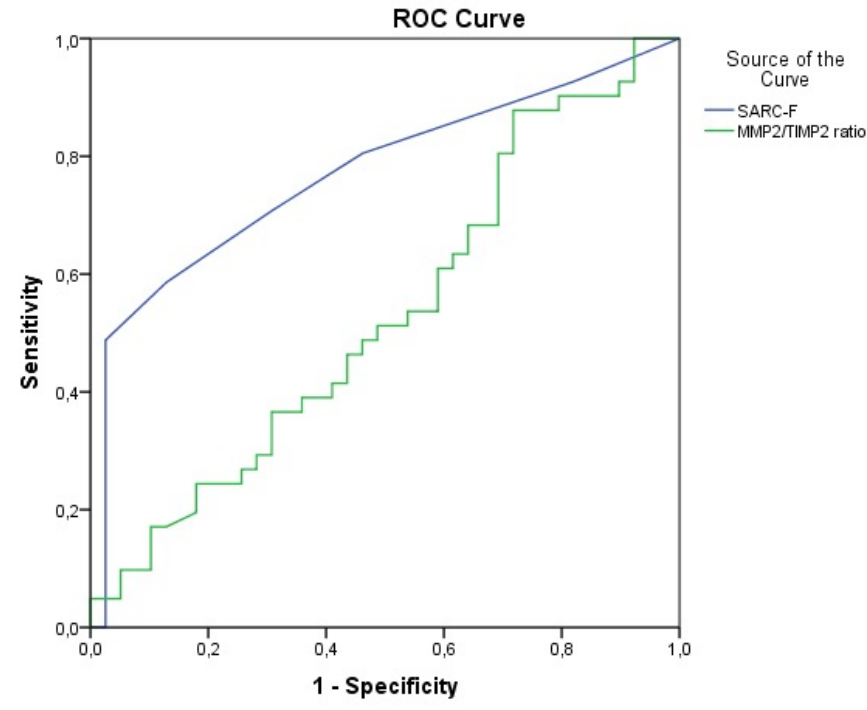

Figure 1. Receiver operating characteristic (ROC) curve analysis for MMP2/ TIMP2 ratio and SARC-F for accuracy of sarcopenia. Area under the ROC curve (AUC) SARC-F $0.771(p<0.001,95 \% \mathrm{Cl} 0.665-0.877)$, MMP2/TIMP2 ratio 0.530 ( $p=0.641,95 \%$ confidence interval $(C I) 0.403-0.658$ ). Sensitivity and specificity for SARC-F score $\geq 4$ were $58.5 \%$ and $87.2 \%$, respectively

was not found to be significant in sarcopenia screening (16). In a study of 1222 elderly Korean individuals, SARC-F was shown to have a high specificity and high negative predictive value (17). In Ida et al's. (18) meta-analysis about SARC-F's accuracy in screening sarcopenia among older adults, screening specifity performance of SARC-F was found to be high, although its sensitivity was poor. Similarly in our study, sensitivity and specifity of SARC-F in screening for sarcopenia were $58.5 \%$ and $87.2 \%$, respectively. Thus we can support the usage of SARC-F questionnaire for screening sarcopenia in elderly, as suggested by EWGSOP2.

Detection of more individuals at risk for sarcopenia led to the need for more practical diagnostic tools. In this study, we investigated the usability of MMP2, TIMP2 levels and MMP2/ TIMP2 ratio as a marker for the diagnosis of sarcopenia in addition to the current diagnostic methods. MMP2, TIMP2 and MMP2/TIMP2 ratio have been studied in many diseases. Both mRNA transcripts and protein expression of MMP2 are reported to be elevated in class-IV lupus nephritis (19). Also in another study MMP2 was found to have a significant role in glioma pathogenesis and could be used as a potential molecular marker for tumor progression (20). Based on the thesis that balance of the MMP/TIMP ratio is important in maintaining the dynamic balance of tissues, its role in lung damage and repair was investigated in the rat study of Chen et al. (21), and it was concluded that lipopolysaccharide-induced acute lung injury may be related to upregulation of MMP2/TIMP2 ratios. Although there are few studies in the literature evaluating the effect of MMP2 and TIMP2 on muscle cells, in a mice study it has been shown that the absence of MMP2 and MT1-MMP causes a significant retardation in myoblast fusion and is incompatible with life due to immature muscle fibers and various organ failures (22). In another study investigating the role of TIMP2 on muscle development and function, it was revealed that TIMP2 was expressed in the neuromuscular junction, regulating neuromuscular junction development and its expression was higher in fast-twitch muscles, thus it has been mentioned that this result can open new horizons in the treatment of conditions such as age-related sarcopenia, in which fast-twitch muscle fibers were lost (23). The number of biomarker studies about sarcopenia is increasing day by day. In Bano et al.'s (24) metaanalysis, evaluating the relationship between sarcopenia and inflammation while patients with sarcopenia had a statistically significantly higher C-reactive protein than control groups, no significant difference was found in terms of tumor necrosis factor-alpha and interleukin-6. In a study comparing sarcopenic and non-sarcopenic control groups, MMP9/TIMP1 ratio was found to be increased in sarcopenic patients, while in another study, sarcopenia was found to be associated with increased FGF-21 and low FGF-19 levels $(25,26)$. In our study, there was no statistically significant difference between sarcopenic and non-sarcopenic groups in terms of serum MMP2, TIMP2 levels and MMP2/TIMP2 ratio. The insignificance of these biochemical markers with sarcopenia may be due to the small sample size, or it may be due to the non-dominance of the inflammatory system in sarcopenia.

There are similarities and interrelationships between sarcopenia and osteoporosis. Many mechanisms have been investigated to explain this relationship. Mechanostat hypothesis, which refers to the muscle contraction action that provides a direct mechanical stimulus to the bone that promotes osteogenesis, the effects of hormones and nutritional state that provide both muscle and bone development, physical activity level, common genetic and developmental components are some of them (5,6,27-29). In a study of two hundred thirty-four women whole-body and femoral neck bone mineral density values were found to be significantly lower in sarcopenia group than non-sarcopenia group (30). In another study, Reiss et al. (31) drew attention to the frequent coexistence of these two conditions in the elderly and associated this with poor function and malnutrition in advanced age. Similarly, the coexistence of osteoporosis and sarcopenia was found to be significant in our study. Because of the relationship between sarcopenia and osteoporosis, our study suggests that these two conditions should be evaluated together.

\section{Study Limitations}

The main limitation of this study is the small sample size and another limitation is that our study was designed as a casecontrol study. 


\section{Conclusion}

Our study supports widespread use of SARC-F questionnaire to screen for risk of sarcopenia. In addition, since the incidence of osteoporosis is significantly higher in sarcopenia, our study supports the evaluation of sarcopenia and bone mineral density together. Another point to be emphasized about the results of our study is that there is a need for other studies investigating the use of serum MMP2, TIMP2 levels and MMP2/TIMP2 ratio for the diagnosis of sarcopenia.

\section{Ethics}

Ethics Committee Approval: This study was approved by the Ethical Review Committee of İstanbul University-Cerrahpaşa, Cerrahpaşa Faculty of Medicine. Ethical Review Committee decision date and number: 2019-22507.

Informed Consent: Informed consent was obtained from the patients.

Peer-review: Externally peer-reviewed.

\section{Authorship Contributions}

Surgical and Medical Practices: V.S., H.Y., I.M.B., D.S.E., Concept: V.S., H.Y., A.D., D.S.E., Design: V.S., B.B.K., A.D., I.M.B., D.S.E., Data Collection or Processing: V.S., B.B.K, I.M.B., D.S.E., Analysis or Interpretation: V.S., I.M.B., A.D., D.S.E., Literature Search: B.B.K., H.Y., A.D., D.S.E., Writing: V.S., B.B.K., A.D., H.Y., D.S.E.

Conflict of Interest: No conflict of interest was declared by the authors.

Financial Disclosure: This work was supported by the Research Fund of İstanbul University-Cerrahpaşa (project number: 33498).

\section{References}

1. Montero-Fernández N, Serra-Rexach JA. Role of exercise on sarcopenia in the elderly. Eur J Phys Rehabil Med 2013;49:131-143.

2. Cruz-Jentoft AJ, Landi F, Topinková E, Michel JP. Understanding sarcopenia as a geriatric syndrome. Curr Opin Clin Nutr Metab Care 2010;13:1-7.

3. Greenlund $\sqcup$, Nair KS. Sarcopenia--consequences, mechanisms, and potential therapies. Mech Ageing Dev 2003;124:287-299.

4. Cruz-Jentoft AJ, Bahat G, Bauer J, Boirie Y, Bruyère O, Cederholm T, Cooper C, Landi F, Rolland Y, Sayer AA, Schneider SM, Sieber CC, Topinkova E, Vandewoude M, Visser M, Zamboni M, Writing Group for the European Working Group on Sarcopenia in Older People 2 (EWGSOP2), and the Extended Group for EWGSOP2. Sarcopenia: revised European consensus on definition and diagnosis. Age Ageing 2019;48:16-31.

5. Karasik D, Kiel DP. Genetics of the musculoskeletal system: a pleiotropic approach. J Bone Miner Res 2008;23:788-802.

6. Frost HM. Bone's mechanostat: a 2003 update. Anat Rec A Discov Mol Cell Evol Biol 2003;275:1081-1101.

7. Nagase $\mathrm{H}$, Woessner JF. Matrix metalloproteinases. J Biol Chem $1999 ; 274: 21491-21494$.
8. Lluri G, Langlois GD, McClellan B, Soloway PD, Jaworski DM. Tissue inhibitor of metalloproteinase-2 (TIMP-2) regulates neuromuscular junction development via a beta1 integrin-mediated mechanism. J Neurobiol 2006;66:1365-1377.

9. Chen $X$, Li Y. Role of matrix metalloproteinases in skeletal muscle: migration, differentiation, regeneration and fibrosis. Cell Adh Migr 2009;3:337-341.

10. El Fahime E, Torrente $Y$, Caron NJ, Bresolin MD, Tremblay JP. In vivo migration of transplanted myoblasts requires matrix metalloproteinase activity. Exp Cell Res 2000;258:279-287.

11. Oh J, Takahashi R, Adachi E, Kondo S, Kuratomi S, Noma A, Alexander DB, Motoda H, Okada A, Seiki M, Itoh T, Itohara S, Takahashi C, Noda M. Mutations in two matrix metalloproteinase genes, MMP-2 and MT1-MMP, are synthetic lethal in mice. Oncogene 2004;23:5041-5048.

12. Cosman F, de Beur SJ, LeBoff MS, Lewiecki EM, Tanner B, Randall S, Lindsay R. National Osteoporosis Foundation. Clinician's Guide to Prevention and Treatment of Osteoporosis. Osteoporos Int 2014;25:2359-2381.

13. Kanis JA, Oden A, Johnell O, Johansson H, De Laet C, Brown J, Burckhardt $P$, Cooper $C$, Christiansen $C$, Cummings $S$, Eisman JA, Fujiwara $S$, Glüer C, Goltzman D, Hans D, Krieg MA, Croix ALA, McCloskey E, Mellstrom D, Melton $\sqcup$, Pols H, Reeve J, Sanders K, Schott AM, Silman A, Torgerson D, van Staa T, Watts NB, Yoshimura N. The use of clinical risk factors enhances the performance of BMD in the prediction of hip and osteoporotic fractures in men and women. Osteoporos Int 2007;18:1033-1046.

14. Zasadzka E, Pieczyńska A, Trzmiel T, Pawlaczyk M. Polish Translation and Validation of the SARC-F Tool for the Assessment of Sarcopenia. Clin Interv Aging 2020;15:567-574.

15. Sánchez-Rodríguez D, Marco E, Dávalos-Yerovi V, López-Escobar J, MessaggiSartor M, Barrera C, Ronquillo-Moreno N, Vázquez-Ibar 0, Calle A, Inzitari M, Piotrowicz K, Duran X, Escalada F, Muniese JM, Duarte E. Translation and Validation of the Spanish Version of the SARC-F Questionnaire to Assess Sarcopenia in Older People. J Nutr Health Aging 2019;23:518-524.

16. Gade J, Beck AM, Rønholt F, Andersen HE, Munk T, Vinther A. Validation of the Danish SARC-F in Hospitalized, Geriatric Medical Patients. J Nutr Health Aging 2020;24:1120-1127.

17. Kim S, Kim M, Won CW. Validation of the Korean Version of the SARC-F Questionnaire to Assess Sarcopenia: Korean Frailty and Aging Cohort Study. J Am Med Dir Assoc 2018;19:40-45.e1.

18. Ida S, Kaneko R, Murata K. SARC-F for Screening of Sarcopenia Among Older Adults: A Meta-analysis of Screening Test Accuracy. J Am Med Dir Assoc 2018;19:685-689.

19. Thiyagarajan D, Fismen S, Seredkina N, Jacobsen S, Elung-Jensen T, Kamper $A L$, Fenton CG, Rekvig OP, Mortensen ES. Silencing of renal DNasel in murine lupus nephritis imposes exposure of large chromatin fragments and activation of Toll like receptors and the Clec4e. PLoS One 2012;7:e34080.

20. Sincevičiūtè $R$, Vaitkienè $P$, Urbanavičiūtè $R$, Steponaitis $G$, Tamašauskas A, Skiriute D. MMP2 is associated with glioma malignancy and patient outcome. Int J Clin Exp Pathol 2018;11:3010-3018.

21. Chen $G, G e D$, Zhu B, Shi H, Ma Q. Upregulation of matrix metalloproteinase 9 (MMP9)/tissue inhibitor of metalloproteinase 1 (TIMP1) and MMP2/TIMP2 ratios may be involved in lipopolysaccharide-induced acute lung injury. J Int Med Res 2020;48:300060520919592.

22. Oh J, Takahashi $R$, Adachi $E$, Kondo $S$, Kuratomi $S$, Noma A, Alexander DB, Motoda H, Okada A, Seiki M, Itoh T, Itohara S, Takahashi C, Noda M. Mutations in two matrix metalloproteinase genes, MMP-2 and MT1-MMP, are synthetic lethal in mice. Oncogene 2004;23:5041-5048.

23. Lluri G, Langlois GD, McClellan B, Soloway PD, Jaworski DM. Tissue inhibitor of metalloproteinase-2 (TIMP-2) regulates neuromuscular junction development via a beta1 integrin-mediated mechanism. J Neurobiol 2006;66:1365-1377. 
24. Bano G, Trevisan C, Carraro S, Solmi M, Luchini C, Stubbs B, Manzato E, Sergi G, Veronese N. Inflammation and sarcopenia: A systematic review and meta-analysis. Maturitas 2017;96:10-15.

25. Suzan V, Yavuzer H, Bag Soytas R, Bektan Kanat B, Arman P, Gedik TE, Unal D, Atar O, Bolayirli IM, Doventas A. The relationship between primary sarcopenia and SARC-F, serum MMP9, TIMP1 levels, and MMP9/TIMP1 ratio in the geriatric patients. Eur Geriatr Med 2021;12:1229-1235.

26. Soytas RB, Suzan V, Arman P, Emiroglu Gedik T, Unal D, Cengiz M, Bolayirli IM, Erdincler DS, Doventas A, Yavuzer H. Association of FGF-19 and FGF-21 levels with primary sarcopenia. Geriatr Gerontol Int 2021;21:959-962.

27. Tritos NA, Biller BM. Growth hormone and bone. Curr Opin Endocrinol Diabetes Obes 2009;16:415-422.
28. Barouki R, Gluckman PD, Grandjean P, Hanson M, Heindel JJ. Developmental origins of non-communicable disease: implications for research and public health. Environ Health 2012;11:42.

29. Edwards MH, Dennison EM, Aihie Sayer A, Fielding R, Cooper C. Osteoporosis and sarcopenia in older age. Bone 2015;80:126-130.

30. Lima RM, de Oliveira RJ, Raposo R, Neri SGR, Gadelha AB. Stages of sarcopenia, bone mineral density, and the prevalence of osteoporosis in older women. Arch Osteoporos 2019;14:38.

31. Reiss J, Iglseder B, Alzner R, Mayr-Pirker B, Pirich C, Kässmann H, Kreutzer $M$, Dovjak P, Reiter R. Sarcopenia and osteoporosis are interrelated in geriatric inpatients. Z Gerontol Geriatr 2019;52:688-693. 